Nota científica

(Short communication)

\title{
PRIMER REGISTRO DE MEGASELIA SP. (DIPTERA: PHORIDAE) COMO PARASITOIDE DE SPHENARIUM PURPURASCENS PURPURASCENS (ORTHOPTERA: PYRGOMORPHIDAE)
}

\author{
FIRST REPORT OF MEGASELIA SP. (DIPTERA: PHORIDAE) \\ AS A PARASITOID OF SPHENARIUM PURPURASCENS PURPURASCENS \\ (ORTHOPTERA: PYRGOMORPHIDAE)
}

\section{Venecia QUESADA-BÉJAR,, ${ }^{1,3}$ Miguel B. NÁJERA-RINCÓN, ${ }^{2,3}$ ENRIQUe REYES-NOVELO ${ }^{4}$ y Carlos E. GONZÁLEZ-ESQUIVEL ${ }^{3, *}$}

\footnotetext{
${ }^{1}$ Campus de Ciencias Biológicas y Agropecuarias, Universidad Autónoma de Yucatán. Apartado Postal 4-116 Mérida Yucatán CP 97100, México <eslabonmichoacan@hotmail.com>.

${ }^{2}$ Campo Experimental Uruapan, Instituto Nacional de Investigaciones Forestales, Agrícolas y Pecuarias. Av. Latinoamericana No. 1101 Col. Revolución C. P. 60500, Uruapan, Michoacán, México<minaj47@hotmail.com>.

${ }^{3}$ Instituto de Investigaciones en Ecosistemas y Sustentabilidad (IIES-Universidad Nacional Autónoma de México), Antigua Carretera a Pátzcuaro No. 8701 Col. Ex Hacienda de San José de La Huerta C.P. 58190 Morelia Michoacán. México.

${ }^{4}$ Centro de Investigaciones Regionales “Dr. Hideyo Noguchi”, Universidad Autónoma de Yucatán, Av. Itzaes por 49 No. 490 Centro. C.P.97000, Mérida, Yucatán, México <enrique.reyes@correo.uady.mx>.

* Autor de correspondencia: <cgesquivel@cieco.unam.mx>.
}

Recibido: 29/04/2016; aceptado: 11/05/2017

Editor responsable: Vicente Hernández Ortiz.

Quesada-Béjar, V., Nájera-Rincón, M. B., Reyes-Novelo, E. y González-Esquivel, C. E. (2017). Primer registro de Megaselia sp. (Diptera: Phoridae) como parasitoide de Sphenarium purpurascens purpurascens (Orthoptera: Pyrgomorphidae). Acta Zoológica Mexicana (n.s.) 33(2), 407-410.

RESUMEN. Se documenta por primera vez la presencia de especies de Megaselia parasitando a Sphenarium purpurascens purpurascens. Se incluyen algunos datos de su biología desde especímenes colectados en agroecosistemas de maíz en la comunidad de Erongarícuaro, Michoacán, México.

El ortóptero más abundante en México es Sphenarium purpurascens (Charpentier, 1842), presentando una distribución muy amplia que comprende trece estados del centro, sur y occidente del país (Márquez-Mayaudón, 1968). Su nombre común es chapulín de la milpa, ya que es abundante en agroecosistemas donde se cultiva maíz (Serrano-Limón \& Ramos-Elorduy, 1989). El principal daño que ocasiona en este cultivo es evitar el llenado
Quesada-Béjar, V., Nájera-Rincón, M. B., Reyes-Novelo, E., \& González-Esquivel, C. E. (2017). First Report of Megaselia sp. (Diptera: Phoridae) as a parasitoid of Sphenarium purpurascens purpurascens (Orthoptera: Pyrgomorphidae). Acta Zoológica Mexicana (n.s.) 33(2), 407-410.

ABSTRACT. The presence of a Megaselia species parasitizing Sphenarium purpurascens purpurascens is documented for the first time. Some data on its biology are included from specimens collected in maize agroecosystems in the municipality of Erongarícuaro, Michoacan, Mexico.

del grano al alimentarse de los estigmas de la mazorca en formación (Tamayo-Mejía, 2009). Sin embargo, este insecto se puede aprovechar como recurso alimenticio, y en la actualidad se vende en mercados de la Ciudad de México y de los Valles Centrales de Oaxaca, donde forma parte de la gastronomía regional (Marcos et al., 2015). Entre julio y diciembre de 2015 se realizaron colectas de ninfas y adultos de Sphenarium p. purpurascens en culti- 
vos de maíz en la localidad de la Ex Hacienda Charahuén (19³1'23"-19³1'13" N y 101²42'32"-101²4'19" W), Erongarícuaro, Michoacán. Los ejemplares colectados fueron trasladados vivos al laboratorio y se confinaron en envases de plástico de un litro de capacidad, cuya tapa fue cubierta con malla fina de $1 \mathrm{~mm}$ de diámetro. Los individuos fueron alimentados con lechuga desinfectada con hipoclorito de sodio al 5\% durante 20 minutos y enjuagada con agua destilada, además de hojuelas de avena como complemento de la dieta. Los individuos muertos fueron colocados inmediatamente en recipientes de plástico de 3.5 x $4.5 \mathrm{~cm}$, cerrados herméticamente en forma de cámara húmeda bajo condiciones de incubación a $27^{\circ}$ C, a los cuales se les adicionó $200 \mu \mathrm{l}$ de agua destilada cada segundo día con el objeto de aislar hongos entomopatógenos. Los individuos parasitados fueron colectados vivos y dadas las condiciones de mantenimiento en el laboratorio se descarta la posibilidad de que los dípteros los colonizaran después de su muerte.

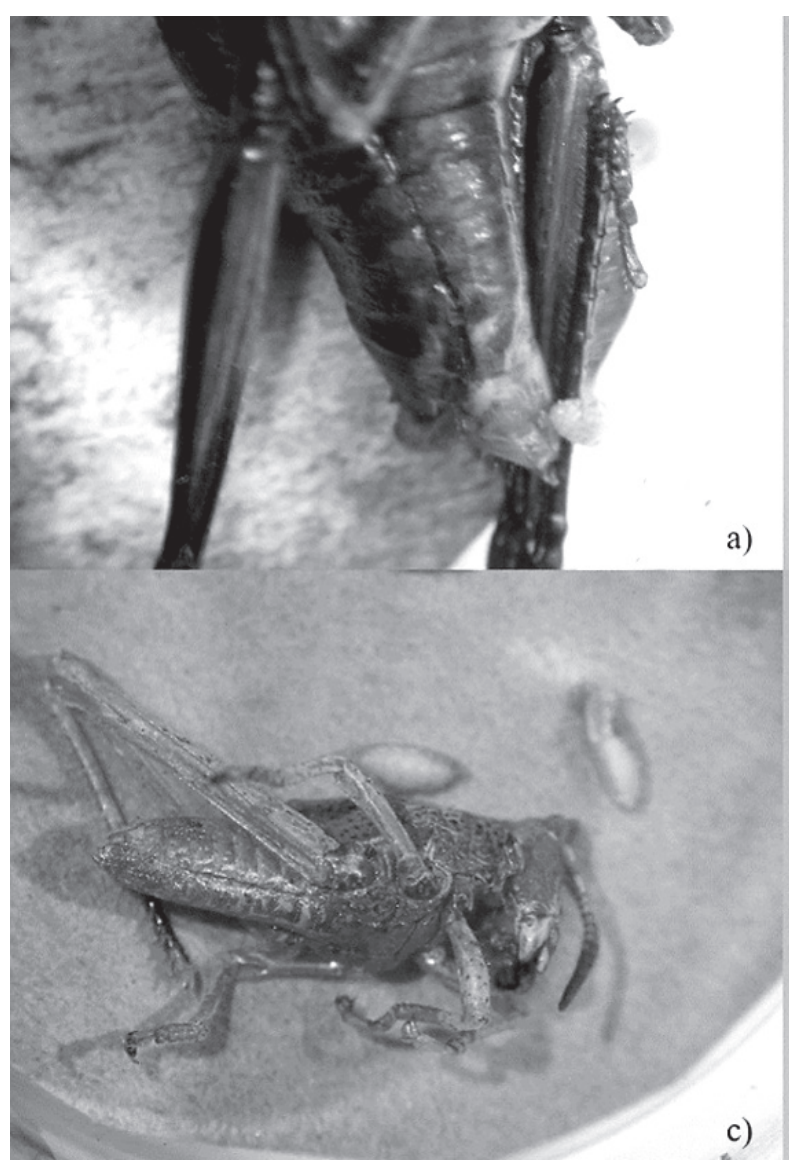

En total fueron recolectados 2,856 individuos de S. p. purpurascens, de los cuales solo 12 especímenes (0.42\%), en su mayoría adultos, presentaron larvas del díptero Megaselia sp. alimentándose en el interior del cuerpo del ortóptero y provocándoles la muerte, por lo que se consideraron una forma de vida parasitoide (Fig. $1)$. Se observó un promedio de emergencia de siete larvas por cada individuo de S. $p$. purpurascens. Nueve de los especímenes parasitados se recolectaron en octubre y tres más en noviembre del 2015 (Cuadro 1). Todas las larvas alcanzaron la pupación, mientras que los dípteros emergidos fueron preservados en alcohol al 70\% y se encuentran depositados en el Laboratorio de Agroecología del Instituto de Investigaciones en Ecosistemas y Sustentabilidad de la Universidad Nacional Autónoma de México (IIESUNAM, Campus Morelia). Los dípteros adultos fueron identificados siguiendo los criterios taxonómicos establecidos en la clave de la familia Phoridae e identificados dentro del género Megaselia Rondani, el cual tiene una dis-
)

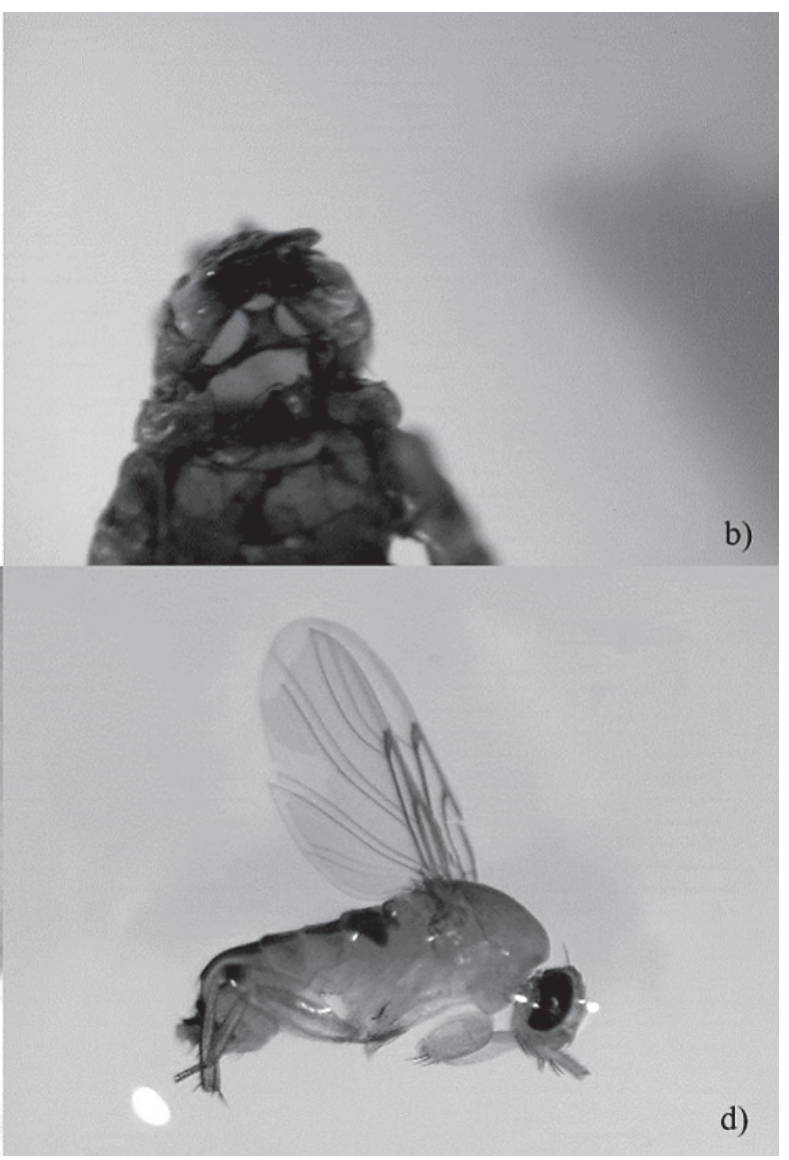

Figura 1. Especímenes de Sphenarium p. purpurascens y sus parasitoides procedentes de cultivos de maíz: a) larvas de Megaselia sp. Emergiendo del abdomen del huésped; b) chapulín consumido por las larvas; c) pupas del parasitoide; d) especímen adulto de Megaselia sp. 
Cuadro 1. Individuos por estadio de desarrollo del hospedero Sphenarium p. purpurascens y sus parasitoides (Megaselia sp.) obtenidos en el laboratorio.

\begin{tabular}{lccc}
\hline & \multicolumn{3}{c}{ Estadio } \\
\cline { 2 - 4 } & Ninfa 3 & Ninfa 4 & Adulto \\
\hline Especímenes parasitados & 2 & 1 & 9 \\
Parasitoides emergidos & 20 & 6 & 59 \\
\hline
\end{tabular}

tribución cosmopolita con aproximadamente 1,500 especies (Peterson, 1987; García-Romera \& Barrientos, 2014).

\section{Megaselia sp.}

Siete especies del género Megaselia han sido registradas en México (Brown, 2017): M. aletiae (Comstock, 1880), M. basispinata (Lundbeck, 1920), M. cordobensis (Malloch, 1912), M. incisa (Malloch, 1912), M. lanceata (Borgmeier, 1962), M. nudipalpis (Borgemeier, 1962) y M. perdita (Malloch, 1912).

Material estudiado: MÉXICO: Michoacán: Erongarícuaro: Ex Hacienda Charahuén: $56 \hat{\jmath}, 29 q$ 우 ex. Sphenarium purpurascens purpurascens, 22-X-2016 y 21-XI-2016.

Comentarios taxonómicos: Este género se caracteriza por tener un par de alas traslúcidas en las que destaca la venación de la parte anterior, venas radiales cortas, engrosadas y concentradas en su base anterior con otras cuatro venas débiles corriendo paralelas en la membrana. La vena Rs generalmente está bifurcada y en algunos casos debilitada; las cavidades antenales son profundas y generalmente bien desarrolladas. La frente presenta uno o dos pares de sedas supra-antenales usualmente más cortas en comparación a las sedas presentes en la parte frontal. El primer flagelómero es redondo u oval y la arista puede estar en posición variable. La probóscide es corta, delgada, poco esclerosada y retráctil (Peterson, 1987).

Comentarios de historia natural. El género Megaselia es el más diverso de la familia Phoridae, ya que posee más de la mitad de las especies de esta familia (GarcíaRomera \& Barrientos, 2014). Este género presenta una gran variedad de hábitos alimenticios, por ejemplo, $M$. halterata (Wood, 1910) se alimenta de hongos, por lo que es considerada una plaga en los cultivos de setas (Navarro et al., 2000). Megaselia lutea (Meigen, 1830) se alimenta de plantas en descomposición (Disney, 1994); los adultos de M. manicata (Wood, 1910) se han registrado como visitantes de flores (Disney, 1994). Por otra parte, M. scalaris (Loew, 1866) contribuye a los procesos de descomposición de cadáveres humanos (Zepeda-Cavazos et al., 2015). Las larvas de M. meconicera (Speiser, 1925) son coprófagas del guano de murciélago (Disney, 1994). Las larvas de M. nasoni (Malloch, 1914) son depredadoras de huevos de Theridiidae (Araneida) y los adultos son visitantes florales de Umbelliferae (Disney, 1994; Disney \& Pagola-Carte, 2009). En México, el conocimiento de la familia Phoridae es muy limitado, con algunos datos sobre el género Megaselia, tales como el registro de individuos de este género polinizando orquídeas en bosques de encino en el estado de Chiapas (Albores-Ortiz \& Sosa, 2006). En depósitos de ámbar se encontraron fósiles de Megaselia sp. que actualmente habitan en los manglares de las zonas costeras (Bown et al., 2005). Se les conoce también como parasitoides facultativos de Comadia redtenbacheri (Lepidoptera: Cossidae) en Tlaxcala, Hidalgo y la Ciudad de México (Hernández-Zetina, 2009). Las larvas de $M$. scalaris se han reportado como parasitoides de Macrodactylus murinus (Coleoptera: Melolonthidae) (Arredondo-Bernal \& Trujillo-Arriaga, 1994) y de la tarántula Brachypelma (Araneae: Theraphosidae) (Machkour-M’Rabet \& Hénaut, 2015).

AGRADECIMIENTOS. Este trabajo forma parte del proyecto SEP-CONACYT 2012-179319, "Importancia de los microbios benéficos de la rizósfera en la producción sustentable de maíz”. VQB agradece al CONACYT la beca de posgrado (444-081). A Virginia Meléndez Ramírez, Luis Abdalá Roberts y Juan Pinzón Esquivel por los comentarios que enriquecieron versiones iniciales de este trabajo.

\section{LITERATURA CITADA}

Albores-Ortiz, O. \& Sosa, V. (2006). Polinización de dos especies simpátricas de Stelis (Pleurothallidinae, Orchidaceae). Acta botánica mexicana, 74, 155-168.

Arredondo-Bernal, H. C. \& Trujillo-Arriaga, J. (1994). Primer reporte de Megaselia scalaris (Diptera: Phoridae) como parasitoide de Macrodactylus murinus (Coleoptera: Scarabaeidae).Vedalia, 1, 27-28.

Barnes, J. K. (1991). Additions to the phorid fauna (Diptera: Phoridae) of North America north of Mexico. Florida Entomologist, 74, 305-310.

Brown, B. V. (2017). Phorid Catalog (PCAT). Online data for phorid flies. Disponible en: http://phorid.net/pcat/index.php (Accesado en febrero 2017).

Disney, R. H. L. (1994). Scuttle Flies: The Phoridae. London: Chapman \& Hall. 196 pp.

Disney, R. H. L. \& Pagola-Carte, S. (2009). Two new species of Megaselia Rondani (Diptera: Phoridae) attracted to bracket fungi (Polyporaceae) in Spain. Heteropterus Revista de Entomología, 9, 87-95. 
García-Romera, C. \& Barrientos, J. A. (2014). La fauna de Phoridae (Diptera) en el Parque Natural del Montseny (Cataluña, España). Citas nuevas para la Península Ibérica. Boletín de la Sociedad Entomológica Aragonesa, 54, 237-261.

Hernández Zetina, D. A. (2009). Parasitoides del gusano rojo del maguey. Tesis de Maestría. Colegio de Postgraduados, Montecillo, México.

Machkour-M'Rabet, S., Dor, A. \& Hénaut, Y. (2015). Megaselia scalaris (Diptera: Phoridae): an opportunistic endoparasitoid of the endangered Mexican redrump tarantula, Brachypelma vagans (Araneae: Theraphosidae). The Journal of Arachnology, 43, 115119.

Marcos, Y. S., Pacheco, R. P., Pérez, G. E. G., Manzanero, G. I. \& Medina, G. R. O. (2015). Conocimiento tradicional y valor cultural de Sphenarium spp., en valles centrales de Oaxaca. Revista Mexicana de Agroecosistemas, 2, 33-35.

Márquez-Mayaudón, C. (1968). Contribución al estudio de los ortópteros de México IV. Ortópteros del Pedregal de San Ángel, Villa Obregón, DF. Anales del Instituto de Biología Serie Zoología, 39, 107-112.

Navarro, M. J., Escudero, A., Gea, F. J., López-Lorrio, A., GarcíaMorrás, J. A. \& Ferragut, F. (2000). Determinación y abundancia estacional de las poblaciones de dípteros (Diptera: Phoridae y Sciaridae) en los cultivos de champiñón en Castilla-La Mancha.
Boletín de Sanidad Vegetal Plagas, 4, 527-536.

Peterson, B. V. (1987). Phoridae. Pp. 689-712. In: J.F. McAlpine, B.V. Peterson, G.E. Shewell, H.J. Teskey, J.R. Vockeroth, \& D.M. Wood (Eds.). Manual of Nearctic Diptera. Vol. 2. Agriculture Canada Monograph No. 28, Canada, 657 pp.

Ruiz-Nájera, R. E., Molina-Ochoa, J., Carpenter, J. E., Espinosa-Moreno, J. A., Ruiz-Nájera, J. A., Lezama-Gutiérrez, R. \& Foster, J. E. (2007). Survey for hymenopteran and dipteran parasitoids of the fall armyworm (Lepidoptera: Noctuidae) in Chiapas, Mexico. Journal of Agricultural and Urban Entomology, 1, 3542.

Serrano-Limón, G. \& Ramos-Elorduy, J. (1989). Biología de Sphenarium purpurascens (Charpentier) y algunos aspectos de su comportamiento (Orthoptera: Acrididae). Anales del Instituto de Biología Serie Zoología, 59, 139-152.

Tamayo-Mejía, F. (2009). Control biológico de Sphenarium purpurascens (Charpentier) y Melanoplus differentialis (Thomas) (Orthoptera: Acrididae) con Metarhizium anisopliae (Metschnikoff) Sorokin, en Guanajuato, México. Vedalia, 13, 85-90.

Zepeda-Cavazos, I. G., Flores-Hernández, G. J., Iruegas-Bientello, F. J., Tijerina-Medina, G., Caballero-Quintero, A. \& Quiroz-Martínez, H. (2015). Diversidad de insectos en necrotrampas expuestas a dos condiciones en el Ojase, Salinas Victoria, Nuevo León, México. Entomología Médica y Forense, 2, 648-654. 